\title{
Metabolomics in Antimicrobial Drug Discovery: The Success of the Chemical Diversity
}

\section{Juan Bueno*}

Bioprospecting Development and Consulting, Bogotá, Colombia

\begin{abstract}
Metabolic networks are an expression of the functionality in biological systems, with the study of metabolomics is possible to obtain data about the physiological state of organisms interacting with various environmental factors, equally develop treatment strategies that reverse the disease. Currently, the metabolomics are a promising tool in drug discovery; because can elucidate drug targets, identify new sources of bioactive compounds and lead the development of new trends for drug production as well as select patients for clinical trials and monitor their health status. In this commentary article will be explored the metabolomics as perhaps the more interesting set of techniques able to influence various fields, and most expectancy to use it to cross the valley of death between basic science and application, especially in a public health threat as is antimicrobial resistance.
\end{abstract}

Keywords: Metabolomics; Metabolites; Antimicrobial; Drug discovery

\section{Introduction}

Metabolomics is an integrative research field that express the functionality of biological systems, through observation of changes in the concentration of metabolites defined as low-molecularweight $(<1 \mathrm{kDa})$ compounds present in metabolic networks [1]. These compounds that belong to metabolic network of functionality configure the metabolome and can include amino acids, nucleotides, fatty acids, cofactors, as well as drugs and drug-associated metabolite [2]. Metabolomics have a broad field of applications in drug discovery both preclinical and clinical development; since compound selection, production optimization, mechanism of action (MoA) until toxicity testing [3]. Also interesting are the applications of metabolomics in biomarker identification, which is possible elucidate the mechanisms involved in disease production, useful in the validation of animal models and selection of patients in clinical trials as well as for assess the clinical efficacy $[4,5]$. Employing these techniques is possible to obtain metabolic profiling that can improve the process of drug candidates selection, and increase the successful rate of translation of animal models to clinical trials because is possible use it for choice the more safety and active option $[5,6]$. In this way, metabolomics is an important tool in new antibiotics discovery, prioritizing the isolation and synthesis of novel molecules, because by conduct multivariate comparisons between active and inactive samples is possible to identify using nuclear magnetic resonance (NMR) techniques the more promising compounds [7]. Equally can determinate the antimicrobial targets of the selected molecules [8]. Also, the isolation and dereplication of new antimicrobial drugs from natural sources is currently a challenge by the large number of compounds detected in the nature, and metabolomics is a powerful tool for data acquisition and analysis that can make the drug discovery process faster [9]. The characteristics of metabolomics and their applications are an important and necessary field in translational research, due can to provide an integrative analysis in all phases of drug development. The aim of this commentary article is explore the metabolomics as perhaps the more interesting set of techniques able to influence various fields, and most expectancy to use it to cross the valley of death between basic science and application.

\section{Metabolomics in Drug Discovery and Development}

The secondary metabolism has produced a broad quantity of molecules and derivate with a great amount of applications in medicine and industry [9]. In this order of ideas, metabolomics methods are useful in bioprospecting of potentially novel antibiotics from biological sources. For identify new chemical entities dereplication studies have used hyphenated methods that couple separation techniques and spectroscopic detection technologies as liquid chromatography-mass spectrometry (LC-MS) and gas chromatography-mass spectrometry (GC-MS), NMR spectroscopy that is a powerful tool in detection and characterization has been introduced in hyphenated methods as LC-NMR and LC-NMR-MS [10], with the end of elucidate the metabolome of microbial extracts as well as natural products from plant or animal sources [11]. Later, the obtained fingerprinting spectra can be introduced in databases for computer assisted structure elucidation (CASE), clustering and classification [10-12]. Finally, natural products databases are available (MarinLit, AntiBase, SciFinder, Beilstein Dictionary of Natural Products and BactiBase) for to give comparative information about biological activity and are useful in compound selection $[13,14]$. Equally the information obtained can be analyzed in functional databases as Kyoto Encyclopedia of Genes and Genomes (KEGG, http://www.genome.jp/kegg/ or http://www.kegg.jp/) that combine genomics with chemical and functionality for acquire a systemic view of molecular interactions [15].

Also, metabolomics have been applied in to increase the biotechnological production and yield of novel drugs [11]. Because metabolomics is an important source of information in the detection and identification of DNA sequences that encode secondary metabolites from natural origins [16]. An interesting approach in this field use synthetic biology technologies that combine biosynthetic pathways knowledge (100 discovered for antibiotics) and genes involved in the synthesis process [17]. These biosynthetic gene clusters, can synthesize

*Corresponding author: Juan Bueno, Bioprospecting development and consulting Bogotá, Colombia, Tel: 571-3108323975; E-mail: juangbueno@gmail.com

Received October 08, 2015; Accepted October 26, 2015; Published November 02, 2015

Citation: Bueno J (2015) Metabolomics in Antimicrobial Drug Discovery: The Success of the Chemical Diversity. J Microb Biochem Technol 7: 380-383. doi:10.4172/1948-5948.1000242

Copyright: (c) 2015 Bueno J. This is an open-access article distributed under the terms of the Creative Commons Attribution License, which permits unrestricted use, distribution, and reproduction in any medium, provided the original author and source are credited. 
a large diversity of compounds with high chemical diversity that can be expressed in microbial strains as the case of Streptomyces lividans, Streptomyces albus, Streptomyces avermitilis and Streptomyces coelicolor for improve the yield of metabolites [18]. Other biotechnological tool employ epigenetic modifiers (DNA methyltransferase and histone deacetylase inhibitors) for control the expression of biosynthetic pathways and increase the generation of biomolecules in fungi $[19,20]$. These epigenetic modifiers have shown the ability of upregulate the production of secondary metabolites encoding in biosynthetic gene clusters [21], which alters the fungal metabolome and opening an important horizon in biotechnology [22].

In addition, metabolomics studies can be employed in measure the metabolic effects of natural molecules with different mechanism of action in cell extracts and biological fluids and obtain a metabolic profile of an organism under controlled experimental conditions, also is possible to identify the metabolites produced under the pharmacological action [23]. Equally, antimicrobial targets for drug discovery in several stages of infection can be determined, for to establish a correlation between biological processes, chemical structure and biological activity [24]. Likewise metabolomics can to discovery new biomarkers for establish conditions of health and sickness [25]. With the ability to evaluate efficacy and toxicity of the novel drugs as well as classify patients enrolled in clinical trials, and determine breakpoints for clinical cure [26]. All this makes metabolomics a discipline able to integrate various stages of development and innovation of new medicines, wherein now can already speak of a preclinical and clinical metabolomics as a novel approach for the pharmaceutical industry.

\section{Metabolomics and High Throughput Screening}

Although High Throughput Screening (HTS) technologies have increased the speed of evaluation and the number of compounds in the experimentation process, not always is possible to select drug candidates, due to the absence of in vitro tests capable of predicting ADMET (absorption, distribution, metabolism, elimination, toxicity) properties [27]. In this lead validation have been used analytical methods as NMR and LC-MS coupled to HTS with the end of study drug-protein interaction as well as cytochrome P450 (CYP450) interactions, increasing the throughput for obtain ADMET profiles of promising compounds $[28,29]$.

On the other hand in antimicrobial screening have been implemented the Biologically Multiplexed Activity Profiling (BioMAP) assay, this bioinformatics integrative platform can give information about antibacterial properties and MoA in a 384 well micro-titer plate format as bioactivity fingerprints with a panel conformed by 15 different strains, that can show comparative profiles of Minimum Inhibitory Concentration (MIC) and perform predictive analysis using BioMAP databases as de-replication strategy; that correlates MICs, microbial strain and MoA from genomic, proteomic and metabolomics data of natural product extract fractions and achieve identify new chemical entities with good agreement with LC-MS and NMR analysis $[13,30]$. This integrative tool is a successful approach in translational science through interdisciplinary.

\section{Metabolomics and Antimicrobial Drug Discovery}

Metabolomics using NMR methods give an efficient, with high accuracy and reproducibility screening platform that can classify new antimicrobial drugs by MoA, becoming an excellent complement to HTS evaluation, in this research field have been demonstrated using a metabolome model of Mycobacterium smegmatis the metabolomic changes associated with antimycobacterial activity as cell wall destruction and inhibition of mycolic acid production. This is an important approach in to increase the possibilities of HTS in select compounds with further in vivo efficacy [31]. Equally, metabolomics have been employed to predict the MoA of many compounds in other microbial models, in fact, antifungal activity and the mechanism of action of Tinospora capillipes extract have been elucidated using liquid chromatography-electrospray ionization-tandem mass spectrometry (LC-ESI-MS) metabolomics analysis, with this method was possible to perform a qualitative analysis of the exometabolome (metabolites that are secreted into fermentation media) [32]. Also metabolomics can classify the different anti-infective substances in relation to their $\mathrm{MoA}$, and discover new targets as well as describe new pharmacological effects in microbial cells [33], very useful for predict drug interactions and design new strategies of combination antimicrobial therapy.

Other interesting approach is study the adaptive capacity of microorganisms in presence of antimicrobials, which is an important subject in prediction of drug resistance and establishes the satisfactory treatment protocols in medical care. For evaluate this, was used a model of Escherichia coli grown in presence of different concentrations of carvacrol, subsequently E. coli endo-metabolome was determined by ${ }^{1} \mathrm{H}$ NMR spectroscopy, leading to the establishment that bacteria increase the metabolism of glucose when it exposed to antibiotics and that this adaptive response changes depending on the dose [23]. Equally, is possible to measure the metabolomics changes produced by antibiotic resistance mutations, so it is possible to establish the degree of adaptation developed and find new biomarkers for the diagnosis of infections caused by multidrug-resistant microorganisms [34].

But perhaps the most interesting field of research due to its practical applications in medicine is the metabolomics of infectious diseases, which seeks to give more detailed information on the physiological condition of the patient infected and breaks new ground in exploring the host pathogen interaction $[35,36]$. For example in the metabolic profiling of patients with sepsis was detected a significant decrease in the levels of lactitol dehydrate and S-phenyl- D -cysteine and an increase in the levels of S-(3-methylbutanoyl)-dihydrolipoamide-E and $\mathrm{N}$-nonanoyl glycine [37]. In tuberculosis (TB) patients plasma, the metabolomics profiles have shown biomarkers as Mycobacterium tuberculosis glycolipids and resolvins, these metabolites differs in upregulation depending if the infectious diseases is active or latent, making it an important biomarker for diagnosis and treatment [38]. Equally, in other studies performed with urine from TB-patients and analyzed by LC-MS, were possible monitor anti-TB therapy and the disease resolution [39]. However, despite their ability of detect, these methods continue to be costly and its implementation in clinical routine will depend on the development of more robust, reproducible and automatable technologies [40].

\section{Toxicometabolomics}

The application of metabolomics in toxicology can provide the metabolome of organisms in direct interaction with the environment and determine the use of an adequate therapy, and predict the adverse effects thereof. For that reason the utilization of toxicometabolomics in drug discovery is a powerful tool both in clinical as industry [41]. In this way, toxicometabolomics look for obtain data about of pathways of toxicity, as well as signatures of toxicity, this information have a broad number of uses in biological sciences [42], because can integrate toxicokinetics and toxicodynamics for create the toxome that have the ability of combine in a multivariate analyses time of exposure, 
chemical structure, biological activity and dose [43]. Finally, with the metabolomics profiles obtained is possible to predict the toxicity response in vitro and in vivo of a specific molecular target or a drug formulation [44].

\section{Conclusions and Perspectives}

Although, metabolomics techniques are powerful tools for drug discovery and diagnosis of infectious diseases, its high cost and complexity in the analysis keeps it away from hospitals and health care, the key for increase robustness and portability of metabolomics methods is the improving of separation techniques, due to they are useful in search and detection of metabolites. In order to solve this issue have been implemented separation methods on the microfluidic format, such as microchip capillary electrophoresis (microchip-CE), which is a miniaturized-LC. This microfluidic device can detect small molecules and metabolites, constituting a new trend in metabolomics microsystems for monitoring, toxicity screening, and clinical diagnostics [45].

Also, the most powerful application of metabolomics in drug discovery is in improve the selectivity process of new drug, as well as describes the in vivo mechanism of action, which is very important for to predict drug interactions and reduce the low success rate in medical application [2].

Other exciting future trend in metabolomics is the use of LCMS and NMR in nanotoxicity, with the end of evaluate the toxicity of nanoparticles and determine the MoA of the nanoformulations involved as well as new nanomaterials [46].

Finally, in natural products research the use of metabolomics in the preparation of chemically engineered extracts (CEEs) is an interesting alternative for improve the activity of known and unknown natural scaffolds. This approach is the start of a new source of future drug leads with high impact using the chemical diversity, that may be a new era of production of new secondary metabolites produced in the laboratory for use in medicine, industry and agriculture [47].

\section{References}

1. Hur M, Campbell AA, Almeida-de-Macedo M, Li L, Ransom N, et al. (2013) A global approach to analysis and interpretation of metabolic data for plant natural product discovery. Nat Prod Rep 30: 565-583.

2. Powers $R$ (2014) The current state of drug discovery and a potential role for NMR metabolomics. J Med Chem 57: 5860-5870.

3. Ramirez T, Daneshian M, Kamp H, Bois FY, Clench MR, et al. (2013) Metabolomics in toxicology and preclinical research. ALTEX 30: 209-225.

4. Denayer T, Stöhr T, Van Roy M (2014) Animal models in translational medicine: Validation and prediction. New Horizons in Translational Medicine 2: 5-11.

5. Kumar B, Prakash A, Ruhela RK, Medhi B (2014) Potential of metabolomics in preclinical and clinical drug development. Pharmacol Rep 66: 956-963.

6. Mak IW, Evaniew N, Ghert M (2014) Lost in translation: animal models and clinical trials in cancer treatment. Am J Transl Res 6: 114-118.

7. Wu C, Choi YH, van Wezel GP (2015) Metabolic profiling as a tool for prioritizing antimicrobial compounds. J Ind Microbiol Biotechnol.

8. Sillerud LO, Larson RS (2012) Advances in nuclear magnetic resonance for drug discovery. Methods Mol Biol 910: 195-266.

9. Breitling R, Ceniceros A, Jankevics A, Takano E (2013) Metabolomics for secondary metabolite research. Metabolites 3: 1076-1083.

10. Gaudêncio SP, Pereira F (2015) Dereplication: racing to speed up the natural products discovery process. Nat Prod Rep 32: 779-810.
11. Tawfike AF, Viegelmann C, Edrada-Ebel $R$ (2013) Metabolomics and dereplication strategies in natural products. Methods Mol Biol 1055: 227-244.

12. Kuhn S, Egert B, Neumann S, Steinbeck C (2008) Building blocks for automated elucidation of metabolites: Machine learning methods for NMR prediction. BMC Bioinformatics 9: 400.

13. Yang JY, Sanchez LM, Rath CM, Liu X, Boudreau PD, et al. (2013) Molecular networking as a dereplication strategy. J Nat Prod 76: 1686-1699.

14. Rocha-Martin J, Harrington C, Dobson AD, O'Gara F (2014) Emerging strategies and integrated systems microbiology technologies for biodiscovery of marine bioactive compounds. Mar Drugs. 12: 3516-3559.

15. Kanehisa M, Goto S, Sato Y, Furumichi M, Tanabe M (2012) KEGG for integration and interpretation of large-scale molecular data sets. Nucleic Acids Res 40: D109-114.

16. Nguyen QT, Merlo ME, Medema MH, Jankevics A, Breitling R, et al. (2012) Metabolomics methods for the synthetic biology of secondary metabolism. FEBS Lett 586: 2177-2183.

17. Wohlleben W, Mast Y, Muth G, Röttgen M (2012) Synthetic biology of secondary metabolite biosynthesis in actinomycetes: Engineering precursor supply as a way to optimize antibiotic production. FEBS Lett 586: 2171-2176.

18. Fedorenko V, Genilloud O, Horbal L, Marcone GL, Marinelli F, et al. (2015) Antibacterial Discovery and Development: From Gene to Product and Back. Biomed Res Int 2015: 591349.

19. Williams RB, Henrikson JC, Hoover AR, Lee AE, Cichewicz RH (2008) Epigenetic remodeling of the fungal secondary metabolome. Org Biomol Chem 6: 1895-1897.

20. Cichewicz RH1 (2010) Epigenome manipulation as a pathway to new natural product scaffolds and their congeners. Nat Prod Rep 27: 11-22.

21. Fisch KM, Gillaspy AF, Gipson M, Henrikson JC, Hoover AR, et al. (2009) Chemical induction of silent biosynthetic pathway transcription in Aspergillus niger. J Ind Microbiol Biotechnol 36: 1199-1213.

22. Lim FY, Sanchez JF, Wang CC, Keller NP (2012) Toward awakening cryptic secondary metabolite gene clusters in filamentous fungi. Methods Enzymol 517: 303-324.

23. Picone G, Laghi L, Gardini F, Lanciotti R, Siroli L, et al. (2013) Evaluation of the effect of carvacrol on the Escherichia coli 555 metabolome by using $1 \mathrm{H}-\mathrm{NMR}$ spectroscopy. Food Chem 141: 4367-4374.

24. Zhang B, Powers R (2012) Analysis of bacterial biofilms using NMR-based metabolomics. Future Med Chem 4: 1273-1306.

25. Johnson SR, Lange BM (2015) Open-access metabolomics databases for natural product research: Present capabilities and future potential. Front Bioeng Biotechnol 3: 22.

26. Beyoğlu D, Idle JR (2013) Metabolomics and its potential in drug development Biochem Pharmacol 85: 12-20.

27. Chen A, Yarmush ML, Maguire T (2012) Physiologically based pharmacokinetic models: Integration of in silico approaches with micro cell culture analogues. Curr Drug Metab 13: 863-880.

28. Yuliana ND, Khatib A, Choi YH, Verpoorte R (2011) Metabolomics for bioactivity assessment of natural products. Phytother Res 25: 157-169.

29. Wu X, Wang J, Tan L, Bui J, Gjerstad E, et al. (2012) In vitro ADME profiling using high-throughput rapidfire mass spectrometry: Cytochrome p450 inhibition and metabolic stability assays. J Biomol Screen 17: 761-772.

30. Wong WR, Oliver AG, Linington RG (2012) Development of antibiotic activity profile screening for the classification and discovery of natural product antibiotics. Chem Biol 19: 1483-1495.

31. Halouska S, Fenton RJ, Barletta RG, Powers R (2012) Predicting the in vivo mechanism of action for drug leads using NMR metabolomics. ACS Chem Biol 7: 166-171.

32. Liu Y, Wen J, Wang Y, Li Y, Xu W (2010) Postulating modes of action of compounds with antimicrobial activities through metabolomics analysis. Chromatographia 71: 253-258.

33. Creek DJ, Barrett MP (2014) Determination of antiprotozoal drug mechanisms by metabolomics approaches. Parasitology 141: 83-92. 
Citation: Bueno J (2015) Metabolomics in Antimicrobial Drug Discovery: The Success of the Chemical Diversity. J Microb Biochem Technol 7: 380383. doi:10.4172/1948-5948.1000242

34. Derewacz DK, Goodwin CR, McNees CR, McLean JA, Bachmann BO (2013) Antimicrobial drug resistance affects broad changes in metabolomic phenotype in addition to secondary metabolism. Proc Natl Acad Sci USA 110: 2336-2341.

35. Serkova NJ, Standiford TJ, Stringer KA (2011) The emerging field of quantitative blood metabolomics for biomarker discovery in critical illnesses. Am J Respir Crit Care Med 184: 647-655.

36. Pacchiarotta T, Deelder AM, Mayboroda OA (2012) Metabolomic investigations of human infections. Bioanalysis 4: 919-925.

37. Su L, Huang Y, Zhu Y, Xia L, Wang R, et al. (2014) Discrimination of sepsis stage metabolic profiles with an LC/MS-MS-based metabolomics approach. BMJ Open Respir Res 1: e000056.

38. Frediani JK, Jones DP, Tukvadze N, Uppal K, Sanikidze E, et al. (2014) Plasma metabolomics in human pulmonary tuberculosis disease: a pilot study. PLoS One 9: e108854.

39. Mahapatra S, Hess AM, Johnson JL, Eisenach KD, DeGroote MA, et al. (2014) A metabolic biosignature of early response to anti-tuberculosis treatment. BMC Infect Dis 14: 53

40. Seymour CW, Yende S, Scott MJ, Pribis J, Mohney RP, et al. (2013) Metabolomics in pneumonia and sepsis: an analysis of the GenIMS cohort study. Intensive Care Med 39: 1423-1434.
41. García-Sevillano MA, García-Barrera T, Navarro F, Abril N, Pueyo C et al. (2015) Combination of direct infusion mass spectrometry and gas chromatography mass spectrometry for toxicometabolomic study of red blood cells and serum of mice Mus musculus after mercury exposure. J Chromatogr B Analyt Technol Biomed Life Sci 985: 75-84.

42. Bouhifd M, Hartung T, Hogberg HT, Kleensang A, Zhao L (2013) Review: toxicometabolomics. J Appl Toxicol 33: 1365-1383.

43. Goncharov NV, Ukolov AI, Orlova TI, Migalovskaia ED, Voitenko NG (2015) Metabolomics: On the way to an integration of biochemistry, analytical chemistry, and informatics. Biology Bulletin Reviews 5: 296-307.

44. van Breemen RB, Fong HH, Farnsworth NR (2008) Ensuring the safety of botanical dietary supplements. Am J Clin Nutr 87: 509S-513S.

45. Kraly JR, Holcomb RE, Guan Q, Henry CS (2009) Review: Microfluidic applications in metabolomics and metabolic profiling. Anal Chim Acta 653: 23-35.

46. Schnackenberg LK, Sun J, Beger RD (2012) Metabolomics techniques in nanotoxicology studies. Methods Mol Biol 926: 141-156.

47. Ramallo IA, Salazar MO, Mendez L, Furlan RL (2011) Chemically engineered extracts: source of bioactive compounds. Acc Chem Res 44: 241-250. 